\title{
Longitudinal analyses of the steroid metabolome in obese PCOS girls with weight loss
}

\author{
Thomas Reinehr ${ }^{1}$, Alexandra Kulle ${ }^{2}$, Juliane Rothermel ${ }^{1}$, Caroline Knop-Schmenn ${ }^{1}$, \\ Nina Lass ${ }^{1}$, Christina Bosse ${ }^{1}$ and Paul-Martin Holterhus ${ }^{2}$ \\ 'Department of Pediatric Endocrinology, Diabetes, and Nutrition Medicine, Vestische Hospital for \\ Children and Adolescents, University of Witten/Herdecke, Datteln, Germany \\ ${ }^{2}$ Division of Pediatric Endocrinology and Diabetes, Department of Pediatrics, University Hospital of \\ Schleswig - Holstein, UKSH, Campus Kiel/Christian Albrechts University of Kiel, CAU, Kiel, Germany
}

Correspondence should be addressed to T Reinehr

Email

T.Reinehr@kinderklinikdatteln.de

\begin{abstract}
Objective: The underlying mechanisms of polycystic ovarian syndrome (PCOS) are not fully understood yet. The aim of the study was to get functional insights into the regulation of steroid hormones in PCOS by steroid metabolomics.

Design: This is a longitudinal study of changes of steroid hormones in 40 obese girls aged $13-16$ years (50\% with PCOS) participating in a 1-year lifestyle intervention. Girls with and without PCOS were matched to age, BMI and change of weight status. Methods: We measured progesterone, 17-hydroxyprogesterone, 17-hydroxyprogenolon, 11-deoxycorticosterone, 21-deoxycorticosterone, deoxycorticosterone, corticosterone, 11-deoxycortisol, cortisol, cortisone, androstenedione, testosterone, dehydroepiandrostendione-sulfate (DHEA-S), estrone and estradiol by LC-MS/MS steroid profiling at baseline and one year later.

Results: At baseline, obese PCOS girls demonstrated significantly higher androstenedione and testosterone concentrations compared to obese girls without PCOS, whereas the other steroid hormones including glucocorticoids, mineralocorticoids, estrogens and precursors of androgens did not differ significantly. Weight loss in obese PCOS girls was associated with a significant decrease of testosterone, androstenedione, DHEA-S, cortisol and corticosterone concentrations. Weight loss in obese non-PCOS girls was associated with a significant decrease of DHEA-S, cortisol and corticosterone concentrations, whereas no significant changes of testosterone and androstenedione concentrations could be observed. Without weight loss, no significant changes of steroid hormones were measured except an increase of estradiol in obese PCOS girls without weight loss. Conclusions: The key steroid hormones in obese adolescents with PCOS are androstenedione and testosterone, whereas glucocorticoids, mineralocorticoids, estrogens and precursors of androgens did not differ between obese girls with and without PCOS.
\end{abstract}

Endocrine Connections (2017) 6, 213-224

\section{Introduction}

Polycystic ovarian syndrome (PCOS) is a heterogeneous syndrome that is characterized by features of anovulation (amenorrhea and oligomenorrhea) combined with symptoms of androgen excess (hirsutism, acne and alopecia) (1). The underlying mechanisms of PCOS are not fully understood yet though obesity and insulin resistance

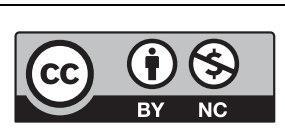

This work is licensed under a Creative Commons Attribution-NonCommercial 4.0 International License. 
are regarded as major risk factors for hyperandrogenism in PCOS due to abnormal sex steroid production in ovaries and adrenals (2). Especially, the differential regulation of precursors of androgens and other steroid hormones within the steroid metabolome encompassing glucocorticoids, mineralocorticoids and estrogens is largely unknown in PCOS.

Although PCOS has frequently been reported in adolescents, the knowledge concerning the disease mechanisms is less clear in adolescents than that in adults due to several reasons $(1,3,4)$ : Longitudinally, studies are rare $(3,5,6)$. Moreover, the diagnosis of PCOS is a challenge in adolescents $(1,3,4)$ as menses irregularities frequently occur in this age range physiologically $(1,3)$. Therefore, it would be useful to have biochemical markers of polycystic ovaries $(3,4)$.

Measurements of steroid hormones such as glucocorticoids, mineralocorticoids and sex hormones are a challenge because of the relatively low specificity of ELISA and RIA. Hence, the state-of-the-art method is a liquid chromatography-tandem mass spectrometry (LC-MS/MS) steroid profiling method (7), which we have used in earlier studies analyzing a comprehensive serum profile of 12 steroid hormones including androgens, mineralocorticoids, glucocorticoids and their precursors in obese prepubertal girls (8). Such data are missing in girls with PCOS.

Therefore, the aim of this study was to identify a PCOS-specific pattern of steroid hormones in adolescents. We analyzed longitudinally a comprehensive profile of 15 steroid hormones including androgens, estrogens, mineralocorticoids, glucocorticoids and their precursors using an LC-MS/MS steroid profiling method (7) in obese adolescent girls with PCOS participating in a lifestyle intervention.

\section{Subjects and methods}

The Local Ethics Committee of the University of Witten/ Herdecke approved this study. Written informed consent was obtained from all subjects and their parents according to the Declaration of Helsinki.

We analyzed a study cohort of 40 obese girls. Ten girls with PCOS were randomly chosen from the successful participants of the Obeldicks intervention (defined by the reduction of their BMI-SDS $>0.5$ during the intervention) (9). Ten girls with PCOS were randomly chosen from the unsuccessful participants of the Obeldicks intervention (defined by increase of their BMI-SDS during the intervention). Twenty girls without PCOS were randomly chosen from the Obeldicks cohort, which were matched to the 20 girls with PCOS according to age, BMI and change of their BMI-SDS.

None of the participants were smokers or took any medication including oral contraceptives.

\section{Definitions}

The diagnosis of PCOS was based on the definition of the Endocrine Society proposed for adolescents (3): Diagnosis included the presence of all of the following: (1) hirsutism, (2) oligo/anovulation and (3) exclusion of other disorders such as androgen-secreting tumors, non-classical adrenal hyperplasia, hyperprolactinemia and thyroid disorders.

Hirsutism was defined as excessive hair distributed in a non-sexual pattern (hair in the face, around the nipples or Tanner pubic hair stage P VI). Oligomenorrhea was defined by menstrual cycles less than every 45 days or less than nine menstrual cycles per year at least 3 years after menarche $(1,10,11)$. Amenorrhea was defined by the absence of cycles $>3$ months at least 3 years after the onset of puberty $(1,10)$.

All girls without PCOS had to demonstrate regular cycle and no hirsutism. Obesity was defined by a BMI for age above the 97th BMI percentile for German adolescents (12). All girls were also obese according to the definition of the International Task Force of Obesity (13).

\section{Measurements}

At baseline and one year later (end-of-lifestyle intervention), steroid hormones (progesterone, 17-hydroxyprogesterone, 17-hydroxyprogenolon, 11-deoxycorticosterone, 21-deoxycorticosterone, deoxycorticosterone, corticosterone, 11-deoxycortisol, cortisol, cortisone, androstenedione, testosterone, dehydroepiandrostenedione sulfate (DHEA-S), estrone (E1), estradiol (E2), luteinizing hormone (LH), folliclestimulating hormone (FSH) and sex-hormone binding globulin (SHBG)) were measured as described below.

Degree of overweight was calculated as body mass index (BMI). We used Box-Cox transformation to calculate BMI-SDS as a measure for degree of overweight (12). As indirect measurement of visceral fat, waist circumference was determined at the narrowest point between the lower rib and the iliac crest during expiration. Triceps and subscapularis skinfold thicknesses were measured twice http://www.endocrineconnections.org DOI: 10.1530/EC-17-0051
() 2017 The authors Published by Bioscientifica Ltd
This work is licensed under a Creative Commons Attribution-NonCommercial 4.0 International License. 
using a caliper and averaged to calculate the percentage of body fat based on the formula of Slaughter (14).

Blood sampling was performed in the fasting status at $8^{\circ}$ in the morning. If the girls were not amenorrheal, the follicular phase was chosen for blood sampling. After clotting, blood samples were centrifuged for $10 \mathrm{~min}$ at $215 \mathrm{~g}$. Serum was stored at $-81^{\circ} \mathrm{C}$ for later determination of steroid hormones. All samples were thawed only once. Serum LH and FSH concentrations were determined by high-specific chemiluminescence immunoassays (ADVIA, Immulite). SHBG was determined by highspecific radioimmunoassays (SHBG-RIACT, cisbio). The intra-assay and interassay coefficient of variation were always $<5.5 \%$. Except DHEA-S and estrogens, all steroid hormones were determined in a parallel assay by LC-MS/ MS as previously described (7). DHEA-S was determined in a separate LC-MS/MS assay previously described (15). E1 and E2 were also measured in a separate LCMS/MS assay with prior derivatization to form dansyl derivatives after extraction. For all assays, $0.1 \mathrm{~mL}$ serum was extracted using Oasis SPE system (Waters, Milford, MA, USA). All steroid measurements were carried out using the same UPLC Quattro Premier/Xe system (Waters). Serum triglyceride, HDL and LDL cholesterol, insulin and glucose concentrations were measured using commercially available test kits (LDL-C, HDL-CPlus Roche Diagnostics; Vitros analyzer Ortho Clinical Diagnostics, Neckargemuend, Germany; MEIA, Abbott). The intra-assay and interassay coefficient of variation were always $<5.5 \%$.

Calculation of free androgen index (AI) was carried out using the formula $100 \times$ testosterone/SHBG (16). Homeostasis model assessment (HOMA) was used to detect the degree of insulin resistance $(\mathrm{HOMA}=$ (insulin $(\mathrm{U} / \mathrm{L}) \times$ glucose $(\mathrm{mmol} / \mathrm{L})) / 22.5(12)$.

\section{Intervention}

The one-year lifestyle intervention 'Obeldicks' has been described in detail elsewhere (9). Briefly, this outpatient intervention program is based on physical activity, nutrition education and behavior therapy including the individual psychological care for the girl and her family.

\section{Statistical methods}

Statistical analyses were carried out with WinSTAT for Exel. Normal distribution was tested by the KolmogorovSmirnov test for all variables. The primary outcome was the comparison calculated by Kruskal-Wallis test of the changes of four key steroid hormones in the four groups: (1) no PCOS and no weight loss, (2) PCOS and no weight loss, (3) no PCOS and weight loss and (4) PCOS and weight loss during the lifestyle intervention. We used five key steroid hormones: testosterone for androgens, cortisol for glucocorticoids, estradiol for estrogens, corticosterone for mineralocorticoids and DHEA-S for precursors of androgens. To account for multiple comparisons, a $P$ value of $<0.01$ was used to define significance.

All further comparisons were performed in explorative nature with a significance level of $P<0.05$. Student $t$-test for paired and unpaired observations, Mann-Whitney $U$ test, Wilcoxon test, Fisher exact test and chi-square test as appropriate. Baseline steroid hormones and their changes were correlated to BMI, BMI-SDS, waist circumference, gonadotropins, insulin and their changes using Spearman correlation. Data are presented as mean \pm S.D., as median and interquartile range (IQR) if variables were not normally distributed or as percentage.

\section{Results}

The baseline study characteristics of the girls are summarized in Table 1. At baseline, all girls were Tanner breast stage B V. All girls with PCOS demonstrated Tanner pubic hair stage P VI at baseline and all girls without PCOS showed Tanner pubic hair stage P V. The girls with PCOS demonstrated significantly higher waist circumference, whereas they did not differ according to BMI or body fat mass. Triglycerides, HOMA, insulin and blood pressure values were significantly higher, whereas the HDL cholesterol concentrations were significantly lower in PCOS girls compared to the girls without PCOS. In the PCOS girls, SHBG concentrations were significantly lower, whereas LH/FSH ratio, AI, LH, androstenedione and testosterone concentrations were significantly higher compared to those in the girls without PCOS. The other steroid hormones did not differ significantly between girls with and without PCOS. However, E2 concentrations were in general lower in girls with PCOS than in those without PCOS.

Comparing the primary outcome, the changes of the five key steroid hormones between the obese PCOS girls with weight loss, the obese PCOS girls without weight loss, the obese non-PCOS girls with weight loss and the obese non-PCOS girls without weight loss demonstrated no significant differences according to glucocorticoids (cortisol), estrogens (E2), mineralocorticoids 
Table 1 Baseline characteristics, cardiovascular risk factors, steroid hormones, sex hormone-binding globulin (SHBG) and gonadotropins in girls with and without PCOS.

\begin{tabular}{l} 
Variable \\
\hline Number \\
Age (years) \\
BMI (kg/m²) \\
BMI-SDS \\
Waist circumference (cm) \\
Triceps skinfold (cm) \\
Subscapularis skinfold (cm) \\
Body fat ${ }^{\text {b }} \%$ ) \\
Triglycerides (mg/dL) \\
HDL-cholesterol (mg/dL) \\
LDL-cholesterol (mg/dL) \\
Fasting glucose (mg/dL) \\
Insulin (U/L) \\
HOMA \\
Systolic blood pressure (mmHg) \\
Diastolic blood pressure (mmHg) \\
LH (U/mL) \\
FSH (U/mL) \\
LH/FSH \\
SHBG (nmol/L) \\
17 Hydroxpregeneolone (nmol/L) \\
Progesterone (nmol/L) \\
17 Hydroxyprogesterone (nmol/L) \\
DHEA-S (nmol/L) \\
Androstenedione (nmol/L) \\
Testosterone (nmol/L) \\
Al \\
E1 (nmol/L) \\
E2 (nmol/L) \\
Deoxycorticosterone (nmol/L) \\
11 Deoxycortisol (nmol/L) \\
21 Deoxycortisol (nmol/L) \\
Corticosterone (nmol/L) \\
Cortisol (nmol/L) \\
Cortison (nmol/L) \\
\end{tabular}

\begin{tabular}{|c|}
\hline PCOS \\
\hline 20 \\
\hline $14.9 \pm 1.1$ \\
\hline $31.2 \pm 3.8$ \\
\hline $2.5 \pm 0.5$ \\
\hline $112 \pm 8$ \\
\hline $33 \pm 5$ \\
\hline $32 \pm 8$ \\
\hline $47 \pm 6$ \\
\hline $137 \pm 43$ \\
\hline 39 (IQR 38-56) \\
\hline $91 \pm 28$ \\
\hline $89 \pm 13$ \\
\hline 23 (IQR 18-34) \\
\hline 4.7 (IQR 3.8-7.8) \\
\hline $125 \pm 14$ \\
\hline $75 \pm 10$ \\
\hline 7.2 (IQR 6.3-10.5) \\
\hline $5.1 \pm 2.4$ \\
\hline 2.0 (IQR 0.9-3.2) \\
\hline 13 (IQR 7-18) \\
\hline 4.1 (IQR 2.6-7.5) \\
\hline 1.0 (IQR 0.4-1.8) \\
\hline $1.1 \pm 0.9$ \\
\hline $3819 \pm 1599$ \\
\hline $4.2 \pm 2.2$ \\
\hline 0.9 (IQR 0.6-1.3) \\
\hline 5.9 (IQR 2.3-13.8) \\
\hline 0.06 (IQR $0.01-0.17$ ) \\
\hline 0.10 (IQR 0.03-0.22) \\
\hline 0.001 (IQR 0.001-0.053) \\
\hline 0.5 (IQR 0.3-1.1) \\
\hline $0.5 \pm 0.3$ \\
\hline 7.4 (IQR 5.0-15.1) \\
\hline 299 (IQR 228-475) \\
\hline $60 \pm 12$ \\
\hline
\end{tabular}

\begin{tabular}{|c|c|}
\hline No PCOS & $P$ value \\
\hline 20 & \\
\hline $14.6 \pm 0.9$ & 0.580 \\
\hline $30.8 \pm 3.0$ & 0.166 \\
\hline $2.3 \pm 0.5$ & 0.528 \\
\hline $103 \pm 10$ & 0.007 \\
\hline $31 \pm 6$ & 0.296 \\
\hline $30 \pm 8$ & 0.304 \\
\hline $43 \pm 7$ & 0.091 \\
\hline $102 \pm 52$ & 0.028 \\
\hline 53 (IQR 44-58) & 0.026 \\
\hline $94 \pm 37$ & 0.686 \\
\hline $89 \pm 8$ & 0.999 \\
\hline 17 (IQR 10-22) & 0.019 \\
\hline 3.9 (IQR 2.0-5.4) & 0.045 \\
\hline $117 \pm 7$ & 0.037 \\
\hline $66 \pm 9$ & 0.007 \\
\hline 3.3 (IQR 2.5-4.2) & $<0.001$ \\
\hline $4.9 \pm 1.8$ & 0.750 \\
\hline 0.7 (IQR 0.6-1.0) & $<0.001$ \\
\hline 25 (IQR 15-35) & 0.007 \\
\hline 4.5 (IQR 2.0-7.7) & 0.897 \\
\hline 1.4 (IQR 0.7-6.6) & 0.184 \\
\hline $1.1 \pm 0.7$ & 0.877 \\
\hline $3480 \pm 1531$ & 0.500 \\
\hline $2.9 \pm 1.0$ & 0.022 \\
\hline 0.5 (IQR 0.2-0.8) & 0.021 \\
\hline 2.1 (IQR 0.6-4.3) & 0.006 \\
\hline 0.07 (IQR 0.05-0.11) & 0.394 \\
\hline 0.17 (IQR 0.10-0.26) & 0.068 \\
\hline 0.001 (IQR 0.001-0.040) & 0.743 \\
\hline 0.5 (IQR 0.4-0.9) & 0.797 \\
\hline $0.5 \pm 0.3$ & 0.263 \\
\hline $6.9(3.7-9.6)$ & 0.358 \\
\hline 244 (IQR 160-329) & 0.070 \\
\hline $57 \pm 12$ & 0.533 \\
\hline
\end{tabular}

Data as mean and S.D. or median and interquartile range, $P$ values derived from unpaired $t$-test or Mann-Whitney $U$ test. Bold indicates significant difference.

${ }^{\mathrm{a}}(100 *$ Test/SHBG); bbased on skinfold thickness.

(corticosterone) and precursors of androgens (DHEA-S), whereas a significant difference in changes of androgens (testosterone) was observed (Fig. 1). Calculating paired comparisons between groups demonstrated that the changes of testosterone in group IV (PCOS and weight loss) differed significantly from the changes of testosterone in group III (no PCOS and weight loss; $P=0.041$ ), from the changes in group II (PCOS and no weight loss; $P=0.008$ ), and from the changes in group I (no PCOS and no weight loss; $P=0.011$ ). The changes of the testosterone concentrations did not differ significantly between the groups I and III calculating paired comparisons. The changes of the other steroid hormones did not differ significantly calculating paired comparison between the four groups (Fig. 1).

\begin{tabular}{|lr}
\hline http://www.endocrineconnections.org & ○ 2017 The authors \\
DOI: 10.1530/EC-17-0051 & Published by Bioscientifica Ltd
\end{tabular}

Weight loss in obese girls with PCOS was associated with a significant decrease in the androgens androstenedione, testosterone and AI values, DHEA-S, cortisol, corticosterone, SHBG and gonadotropins (LH and LH/FSH), whereas all other steroid hormones did not change significantly (Table 2). Without weight loss, estradiol increased significantly in obese PCOS girls, whereas all other steroid hormones did not change significantly. At baseline, the steroid hormones did not differ significantly between obese PCOS girls with and without weight loss.

Analyzing only obese girls without PCOS showed that those with weight loss demonstrated a significant increase in SHBG and a significant decrease in DHEA-S, corticosterone and cortisol concentrations, whereas all

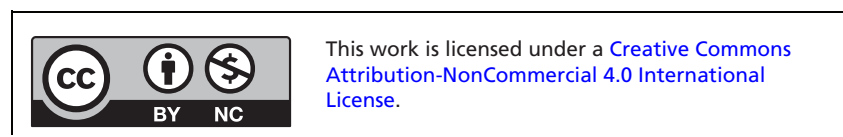




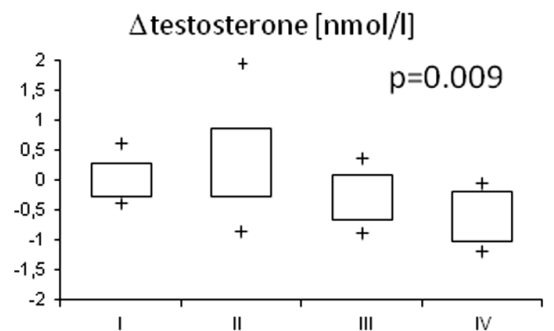

$\Delta$ corticosterone $[\mathrm{nmol} / \mathrm{l}]$

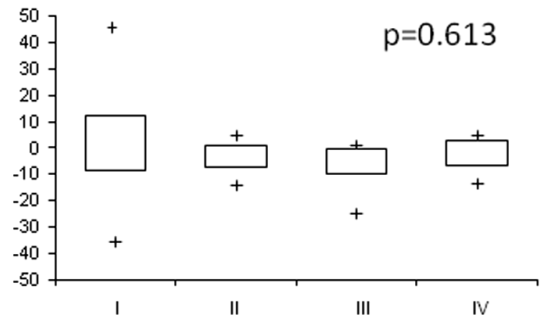

$\Delta$ estradiol $[\mathrm{nmol} / \mathrm{l}]$

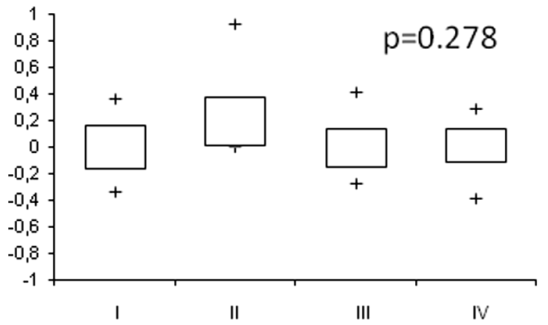

$\triangle$ DHEAS $[\mathrm{nmol} / \mathrm{l}]$

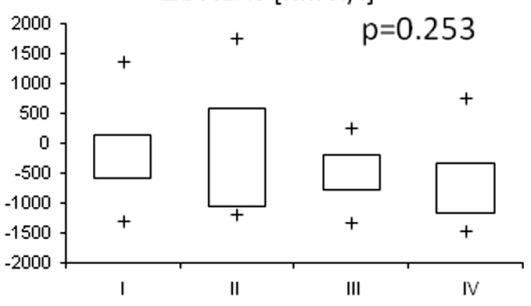

$\Delta$ cortisol $[\mathrm{nmol} / \mathrm{l}]$

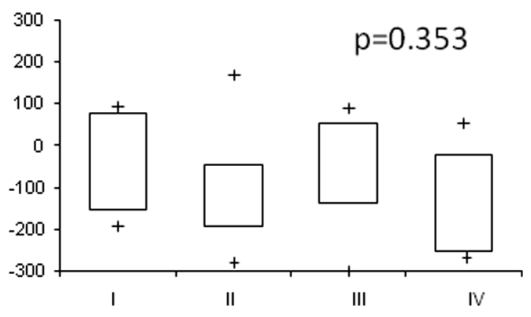

I: no PCOS \& no weight loss

\section{II: PCOS \& no weight loss}

III: no PCOS \& weight loss

\section{IV: PCOS \& weight loss}

\section{$\Delta$ : variable at $1 y$ follow-up - variable at baseline}

\section{Figure 1}

Changes $(\Delta)$ of testosterone, DHEA-S, estradiol, cortisol and corticosterone in 40 obese girls separated to change of weight status and the diagnosis of PCOS (box plots, $P$ values derived from Kruskal-Wallis tests). other steroid hormones including androstenedione and testosterone did not change significantly (Supplementary Table 1, see section on supplementary data given at the end of this article). The obese non-PCOS girls without weight loss demonstrated no significant changes of any steroid hormone. At baseline, the obese non-PCOS girls with and without weight loss did not differ concerning any steroid hormone or cardiovascular risk factor.

Weight loss in obese girls with and without PCOS was associated with a significant decrease of body fat, waist circumference, insulin, HOMA and blood pressure values as well as a significant increase of HDL cholesterol concentrations, whereas an increase of BMI-SDS in the observation period was not associated with any significant improvement of cardiovascular risk factors except a decrease of insulin and HOMA in PCOS girls (Table 2 and Supplementary Table 1). The obese girls with and without weight loss did not differ significantly at baseline according to any cardiovascular risk factor, age or body composition.

The associations between baseline BMI, insulin, gonadotropins, body fat, waist circumference and steroid hormones calculated in the entire study cohort

$\begin{array}{lr}\text { http://www.endocrineconnections.org } & \text { ○ } 2017 \text { The authors } \\ \text { DOI: } 10.1530 / \text { EC-17-0051 } & \text { Published by Bioscientifica Ltd }\end{array}$

are demonstrated in Table 3. Free androgen index was significantly positively and SHBG was negatively related to BMI, insulin and waist circumference in girls with PCOS. Furthermore, testosterone correlated with waist circumference and insulin in girls with PCOS. These significant associations were not observed in girls without PCOS.

The associations between changes of BMI, insulin, gonadotropins, body fat, waist circumference and changes of steroid hormones are demonstrated in Table 4. Changes of testosterone and AI were significantly associated with changes of insulin, BMI, BMI-SDS and waist circumference in girls with PCOS but not in girls without PCOS. Changes of DHEA-S were significantly related to changes of insulin in girls with PCOS. Changes of SHBG were negatively correlated to changes of insulin, waist and BMI in both girls with and without PCOS.

\section{Discussion}

To the best of our knowledge, this is the first study analyzing the longitudinal relationships between a comprehensive steroid profile encompassing androgens and their

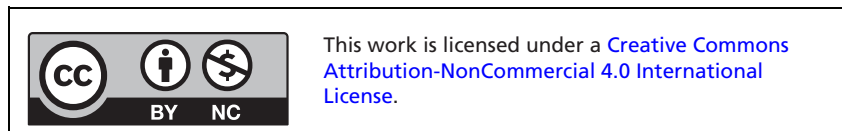


$\frac{\pi}{\frac{\pi}{2}}$

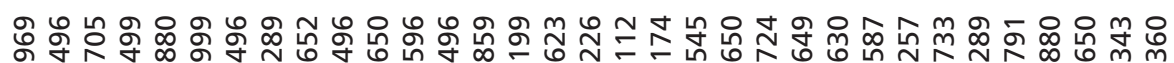

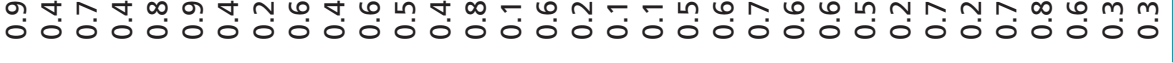

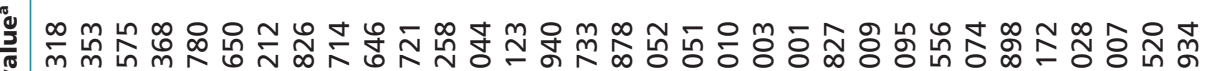

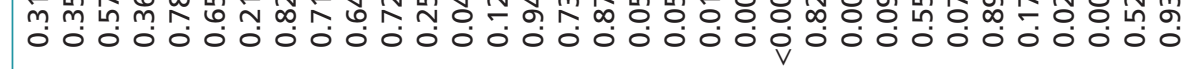

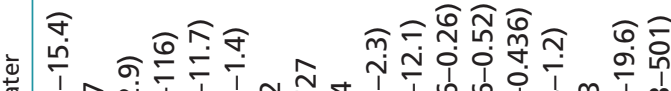
荡

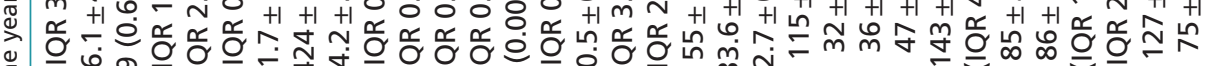
o. -

(1)

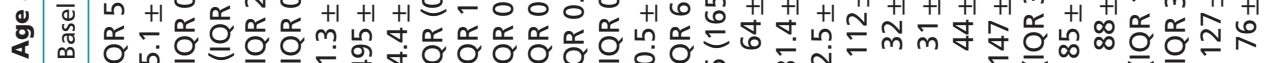

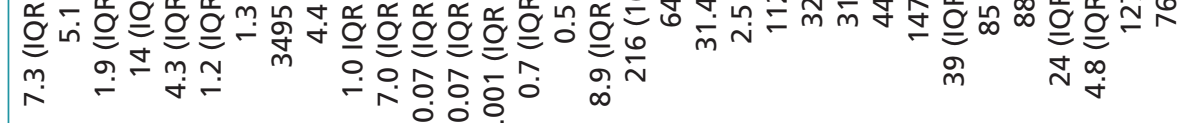

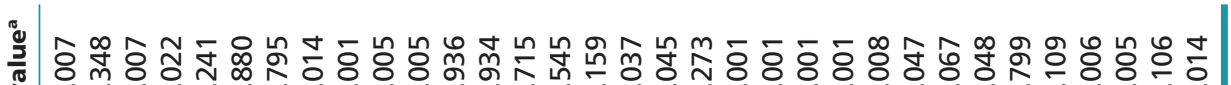

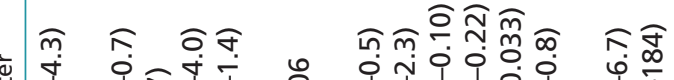

莎

$\simeq$

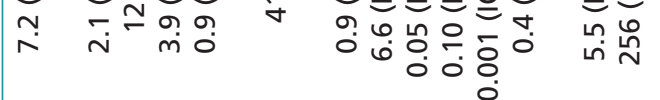
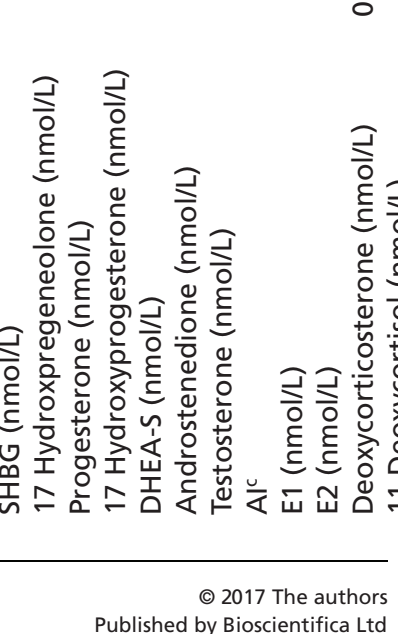


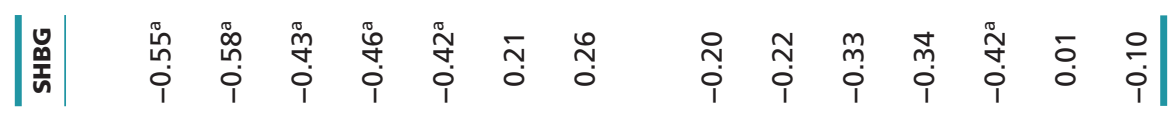

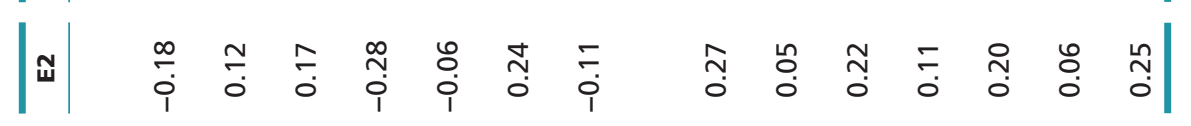

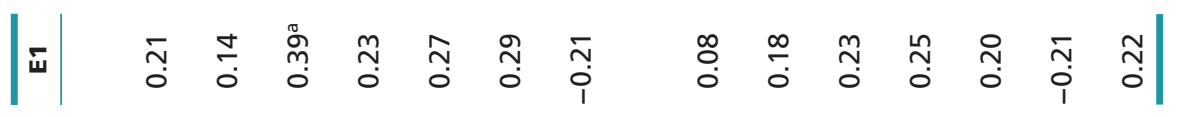

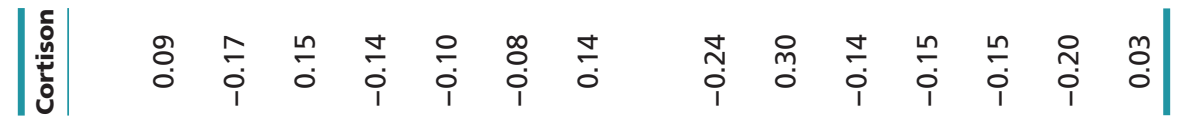

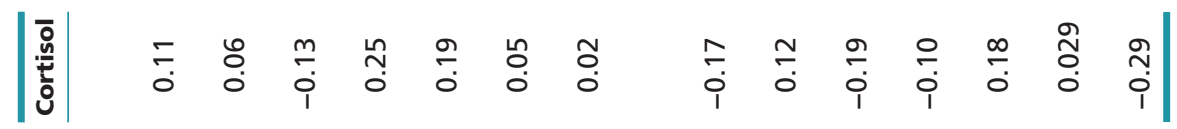

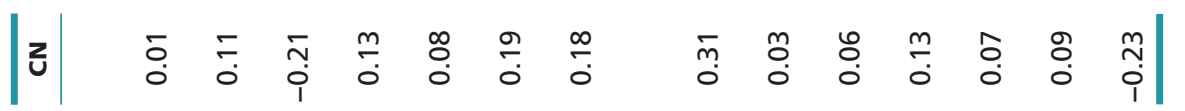

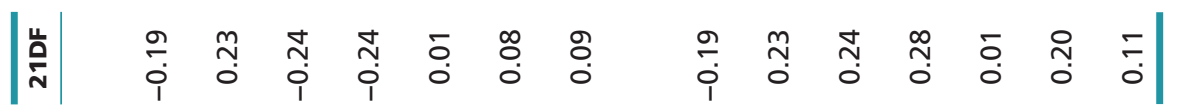

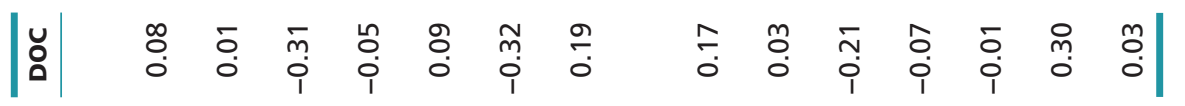

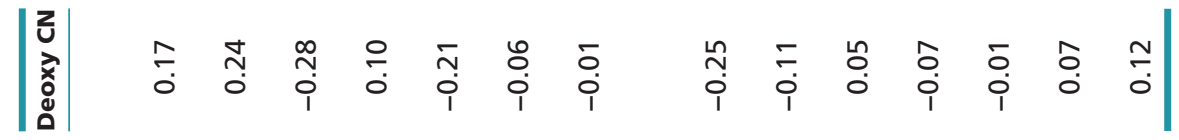

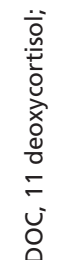

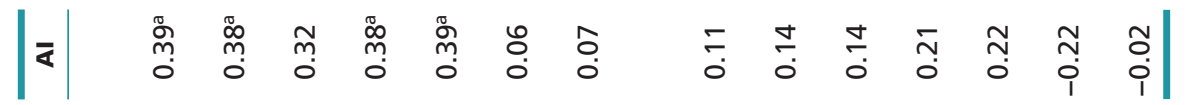

过

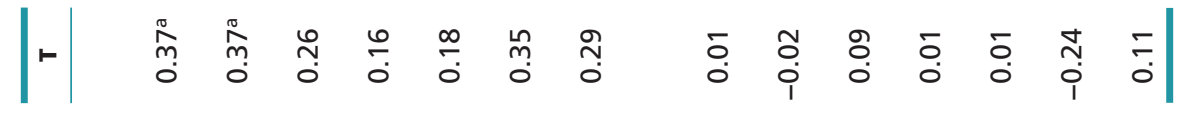

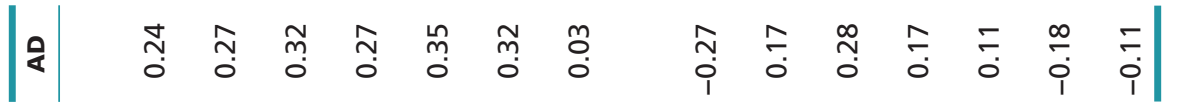

齐

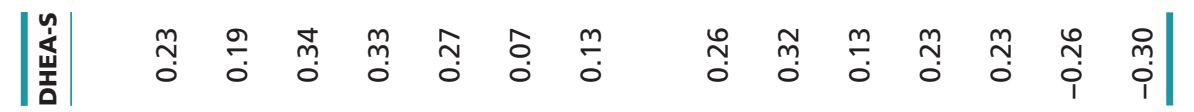

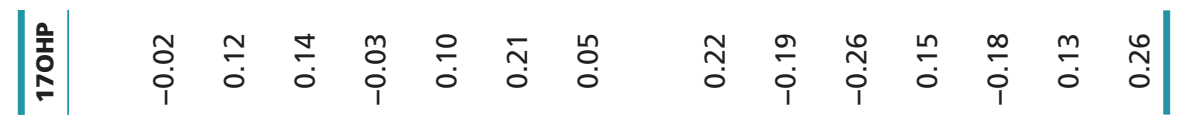

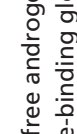

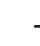

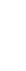

.

.

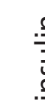

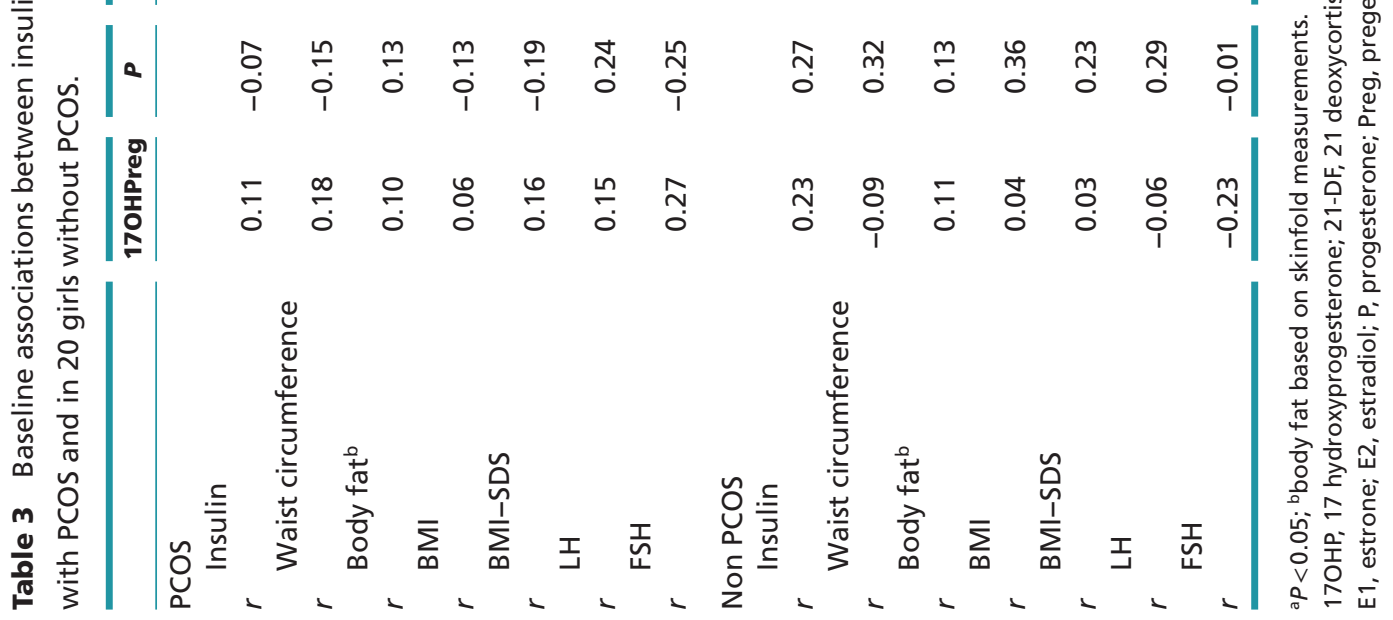




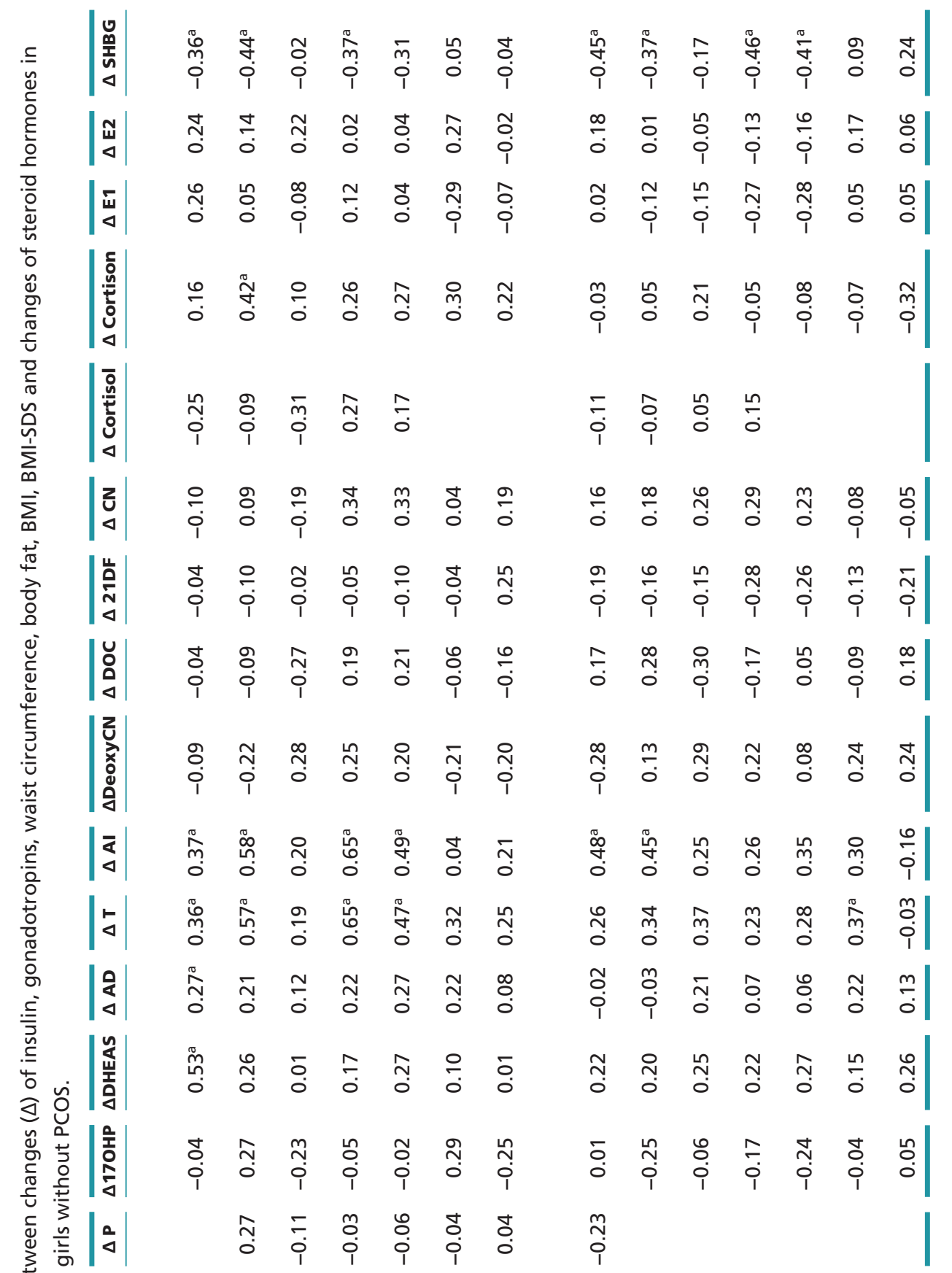

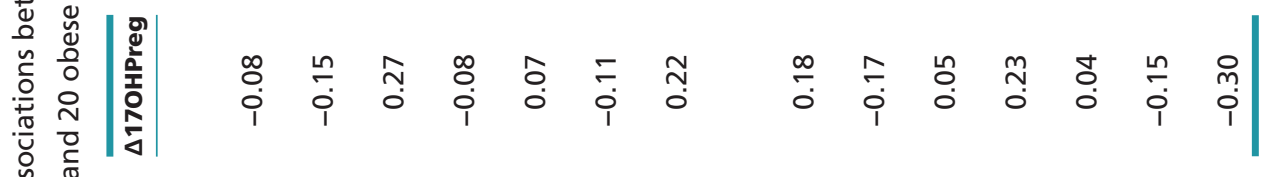

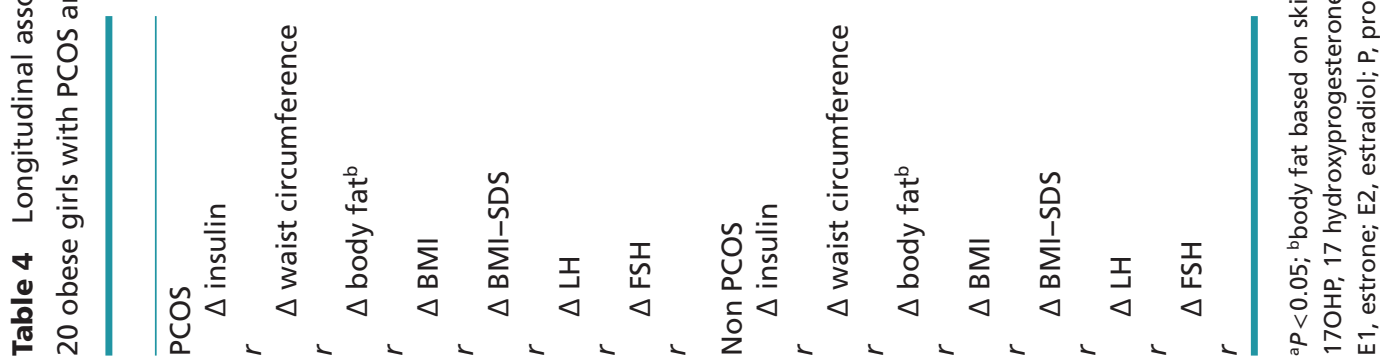

http://www.endocrineconnections.org DOI: 10.1530/EC-17-0051
() 2017 The authors Published by Bioscientifica Ltd

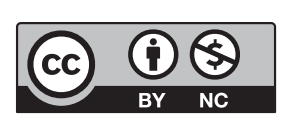

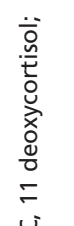<smiles>[Li][Mg]</smiles> 
precursors, estrogens, glucocorticoids, mineralocorticoids as well as body composition, gonadotropins and insulin resistance index HOMA in obese girls with and without PCOS participating in a lifestyle intervention. The main differences in respect to the steroid metabolome were higher androgens in PCOS girls, which normalized in weight loss, whereas estrogens, glucocorticoids, mineralocorticoids and precursors of androgens did not differ between obese girls with and without PCOS. These findings suggest multiple mechanisms establishing a PCOS phenotype in obese girls.

\section{Androgens}

As expected to the definition of PCOS, androstenedione and testosterone concentrations as well as $\mathrm{AI}$ values were higher in obese PCOS girls compared to obese girls without PCOS in our study. These findings are in line with previous studies in adolescents $(5,6,17)$ and women $(18,19)$. Furthermore, these androgens decreased only in the obese PCOS girls with weight loss. Most importantly, we were able to demonstrate for the first time in a longitudinal study of obese adolescents that the precursors of androstenedione and testosterone did not differ between the obese girls with and without PCOS. These findings support the hypotheses of an increased activity of the $3 \beta$-hydroxysteroid dehydrogenase (3ßHSD) and/or the 17-beta-hydroxysteroiddehydrogenase (17 $\beta$ HSD) in PCOS. Insulin stimulates synergistically with $\mathrm{LH}$, the $17 \beta \mathrm{HSD}$ enzyme in vitro, which is the limiting enzyme of androgen synthesis (20). Accordingly, insulin concentrations were related to androgens both in crosssectional and longitudinal analyses in the girls with PCOS in our study.

Insulin also inhibits hepatic synthesis of SHBG (21), the key circulating protein that binds to testosterone, and thus, increases the proportion of AI. In our study, insulin was negatively related to SHBG both in cross-sectional and longitudinal analyses of girls with PCOS.

Of interest, the DHEA-S concentrations in the obese adolescents with and without PCOS were two times higher in our study compared to normal-weight girls of the same age using the same method (median DHEA-S, $2774 \mathrm{nmol} / \mathrm{L})(8,15)$. This finding is in concordance with previous studies in obese adults (22). Although we have previously reported stable DHEA-S concentrations in prepubertal obese children aged $6-8$ years with weight loss (8), we found a decrease in DHEA-S concentrations in the obese girls with weight loss regardless of whether they have
PCOS or not. This apparent discrepancy can be explained by the ongoing adrenarche in our previous younger study cohort leading to higher DHEA-S concentrations outbalancing the depressing effect on DHEA-S by weight loss (23). An increased DHEA-S production due to elevated 17,20 -lyase activity has been reported in obesity $(15,24)$. The adipokine leptin has a documented dose-dependent role to stimulate 17,20-lyase (25).

The increase of DHEA-S in obesity (or in other conditions such premature adrenarche) might serve as a biochemical resource for androgen production in PCOS. Recent research points toward desulfation of DHEA-S to dehydroepiandrosterone (DHEA), which is the base for subsequent androgen biosynthesis (26). An important enzymatic step for androgen formation is the $\Delta 5$ to $\Delta 4$ conversion of DHEA to androstenedione. It has been shown that the $3 \beta \mathrm{HSD}$ expression can be enhanced via an insulin-receptor substrate (IRS) 1- and 2-dependent pathway in different tissue types (27). Therefore, the elevated insulin concentrations in obesity could activate this enzymatic step, thus contributing to the observed increased androstenedione concentrations in PCOS.

The hypothesis that increased DHEA-S in obesity might 'feed' a disturbed pathway of desulfation and subsequent androgen biosynthesis in PCOS would explain the reported association between premature adrenarche and PCOS later in life (28). In this line, bilateral adrenal hyperplasia has been reported as a possible mechanism for hyperandrogenism in women with PCOS (29). Furthermore, corticotrophin-releasing hormone has been suggested to increase adrenal androgen secretion in children (30).

\section{Glucocorticoids}

It is well known that obese girls had higher cortisol concentrations compared to normal-weight girls (8). In concordance, the cortisol concentrations in the obese girls with and without PCOS in our study were two times higher compared to normal-weight girls of the same age using the same method (median cortisol, $160 \mathrm{nmol} / \mathrm{L}$ ) (31). The finding of increased cortisol concentrations in PCOS girls compared to normal-weight girls is in contrast to a study of urine steroid analyses reporting 21-hydroxylase insufficiency in PCOS girls (32). However, this study was small consisting of only 7 girls with PCOS, and the diagnostic criteria of PCOS were not fulfilled in all girls (32). In our study, weight loss was associated with a decrease of cortisol concentrations according to previous 
studies (8) regardless of whether the girls had PCOS or not. These findings support the hypotheses that the adrenal stimulation is attributed primarily to obesity and is not a specific phenomenon seen only in PCOS.

\section{Estrogens}

Our data demonstrated in tendency lower E2 concentrations in girls with PCOS compared to girls without PCOS even if the girls with PCOS had higher androgens. This finding supports the hypothesis of a decreased aromatase activity in girls with PCOS (33). Estrogens are transformed from androgens by aromatase. Experimental studies in animal models revealed that antiMuellerian hormone (AMH), which is increased in PCOS (34), inhibits the FSH-dependent aromatase in granulosa cells (35). Studies in women with PCOS reported negative associations between $\mathrm{AMH}$ and estradiol concentrations (36). As in addition, E1, which is produced predominately in the fat tissue (37), was not higher in the obese PCOS girls with increased androgens compared to obese girls without PCOS and lower androgens, this finding suggests a decreased aromatase activity not only in the ovaries but also in the fat tissue. The concentrations of estradiol, the most active estrogen of ovarian origin with the highest concentration in humans, increased in our study over time in PCOS girls, who did not lose weight. This finding suggests that higher estradiol concentrations do not occur at the beginning of the disease but later in the course of PCOS when increased androgen concentrations may overcome aromatase deficiency. In concordance, it is well known that estradiol concentrations are increased in women in the course of PCOS (2).

\section{Mineralocorticoids}

The predominant feature of the mineralocorticoid pattern reported in obese prepubertal children was an elevation of corticosterone, which dropped significantly after weight loss approximately to the range seen in normal-weight children (8). This drop of corticosterone concentrations was also seen in our study in weight loss regardless of whether the girls were suffering from PCOS or not. Therefore, the observed changes of mineralocorticoids were not specific for PCOS.

\section{Impact of body composition on PCOS}

Interestingly, waist circumference was stronger associated with testosterone than total body fat in our study both in cross-sectional and in longitudinal analyses. Because waist circumference is an indirect parameter for visceral fat mass, our results are in line with the hypothesis that visceral and not total body fat is the main driver of PCOS development in obese adolescent girls (38). Accordingly, Ibanez and coworkers have demonstrated an increased visceral fat mass in normal-weight adolescent girls with PCOS (38). Of interest, visceral fat mass differs in their expression of adipocytokines from subcutaneous fat mass, which may contribute to changes of $17 \beta \mathrm{HSD}$ and $3 \beta \mathrm{HSD}$ in PCOS. For example, resistin is secreted primarily in visceral fat and leads to a follicle arrest (37).

\section{Strengths and limitations of the study}

The strengths of this study are its longitudinal design and the analyses of a comprehensive profile of steroids by a LC-MS/MS multisteroid profiling method (7). Furthermore, studies in adolescents are important as adverse patterns of PCOS itself originate in adolescence $(2,39)$. A further advantage of examining adolescent girls is that there is usually no potential confusion with other diseases or medication.

However, our study presents some potential limitations. First, BMI and skinfold measurements are only indirect parameters of body fat. Second, waist circumference is also only an indirect parameter of visceral fat. Third, we were not able to distinguish the follicular from the luteal phase in the amenorrheal PCOS girls probably influencing the results. We have to keep in mind that a single measurement of LH and FSH as in our study provides little diagnostic sensitivity as gonadotropin concentrations are released in a pulsatile fashion into the circulation and vary the menstrual cycle. Fourth, the diagnosis of PCOS is a challenge in adolescents $(2,40)$. Menses irregularities frequently occur in this age range physiologically. However, the age and symptoms of our patients were similar to adolescent girls suffering from PCOS reported in the literature. Fifth, we have not analyzed the degree of hirsutism before and after lifestyle intervention. However, hirsutism grading in young girls is hard and influenced by subjectivity. Furthermore, no established scores for adolescents are available (3). Sixth, the study sample was only moderate and one might argue that also greater weight loss may lead to further significant changes of steroid hormones. However, the study sample was sufficient to demonstrate a significant change of androgens in PCOS girls with weight loss. Finally, our analyses were determined only in obese adolescents,

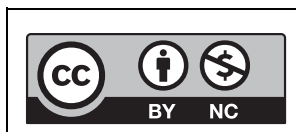

This work is licensed under a Creative Commons Attribution-NonCommercial 4.0 International License. 
whereas PCOS has also reported in lean adolescents (38) suggesting further mechanisms leading to PCOS.

In summary, our analyses of the steroid metabolome demonstrated that the key steroid hormonal parameters in PCOS of obese adolescents are reversible increased androgens such as androstenedione and testosterone, whereas there is no difference in glucocorticoids, mineralocorticoids and precursors of androgens between obese girls with and without PCOS. Future research is needed to understand the specific steroid hormone profile in PCOS in obese and lean adolescents.

\section{Supplementary data}

This is linked to the online version of the paper at http://dx.doi.org/10.1530/ EC-17-0051.

\section{Declaration of interest}

The authors declare that there is no conflict of interest that could be perceived as prejudicing the impartiality of the research reported.

\section{Funding}

Thomas Reinehr received a grant from the European Union's Seventh Framework Programme ( $n^{\circ}$ 115372). Furthermore, Thomas Reinehr and Paul Martin Holterhus received a grant from Ferring. The research leading to these results has received support from the Innovative Medicines Initiative Joint Undertaking under EMIF grant agreement $n^{\circ}$ 115372 , resources of which are composed of financial contribution from the European Union's Seventh Framework Programme (FP7/2007-2013) and EFPIA companies' in kind contribution. This manuscript does not necessarily reflect the views of the Commission and in no way anticipates the future policy in this area. Further grant support by Ferring is gratefully acknowledged. The funders had no role in study design, data collection and analysis, decision to publish or preparation of the manuscript.

\section{Author contribution statement}

Thomas Reinehr and Paul Martin Holterhus developed the study design. Christina Bosse, Caroline Knop-Schmenn, Juliane Rothermel and Nina Lass performed the clinical measurements and Alexandra Kulle obtained the laboratory measurements. Thomas Reinehr performed the statistical analyses. Thomas Reinehr wrote the first draft of the paper. All authors discussed the findings. Each author listed on the manuscript has seen and approved the submission of this version of the manuscript and takes full responsibility for the manuscript.

\section{Acknowledgements}

The authors thank the participating girls of this study.

\section{References}

1 Franks S. Polycystic ovary syndrome in adolescents. International Journal of Obesity 200832 1035-1041. (doi:10.1038/ijo.2008.61)

2 Ehrmann DA. Polycystic ovary syndrome. New England Journal of Medicine 2005352 1223-1236. (doi:10.1056/NEJMra041536)

3 Legro RS, Arslanian SA, Ehrmann DA, Hoeger KM, Murad MH, Pasquali R, Welt CK and Endocrine Society. Diagnosis and treatment of polycystic ovary syndrome: an endocrine society clinical practice guideline. Journal of Clinical Endocrinology and Metabolism 201398 4565-4592. (doi:10.1210/jc.2013-2350)

4 Witchel SF, Oberfield S, Rosenfield RL, Codner E, Bonny A, Ibanez L, Pena A, Horikawa R, Gomez-Lobo V, Joel D, et al. The diagnosis of polycystic ovary syndrome during adolescence. Hormone Research in Paediatrics 201583 376-389. (doi:10.1159/000375530)

5 Lass N, Kleber M, Winkel K, Wunsch R \& Reinehr T. Effect of lifestyle intervention on features of polycystic ovarian syndrome, metabolic syndrome, and intima-media thickness in obese adolescent girls. Journal of Clinical Endocrinology and Metabolism 201196 3533-3540. (doi:10.1210/jc.2011-1609)

6 Hoeger K, Davidson K, Kochman L, Cherry T, Kopin L \& Guzick DS. The impact of metformin, oral contraceptives, and lifestyle modification on polycystic ovary syndrome in obese adolescent women in two randomized, placebo-controlled clinical trials. Journal of Clinical Endocrinology and Metabolism 200893 4299-4306. (doi:10.1210/jc.2008-0461)

7 Kulle AE, Riepe FG, Melchior D, Hiort O \& Holterhus PM. A novel ultrapressure liquid chromatography tandem mass spectrometry method for the simultaneous determination of androstenedione, testosterone, and dihydrotestosterone in pediatric blood samples: ageand sex-specific reference data. Journal of Clinical Endocrinology and Metabolism 201095 2399-2409. (doi:10.1210/jc.2009-1670)

8 Reinehr T, Kulle A, Wolters B, Lass N, Welzel M, Riepe F \& Holterhus PM. Steroid hormone profiles in prepubertal obese children before and after weight loss. Journal of Clinical Endocrinology and Metabolism 201398 E1022-E1030. (doi:10.1210/jc.2013-1173)

9 Reinehr T, Kleber M, Lass N \& Toschke AM. Body mass index patterns over $5 \mathrm{y}$ in obese children motivated to participate in a 1-y lifestyle intervention: age as a predictor of long-term success. American Journal of Clinical Nutrition 201091 1165-1171. (doi:10.3945/ ajcn.2009.28705)

10 Diaz A, Laufer MR \& Breech LL. Menstruation in girls and adolescents: using the menstrual cycle as a vital sign. Pediatrics $2006 \mathbf{1 1 8}$ 2245-2250. (doi:10.1542/peds.2006-2481)

11 Hardy TS \& Norman RJ. Diagnosis of adolescent polycystic ovary syndrome. Steroids $2013 \mathbf{7 8} 751-754$. (doi:10.1016/j. steroids.2013.04.011)

12 German Obesity Association. Guidelines of the German working group on obese children and adolescents. Berlin, Germany: Arbeitsgemeinschaft Adipositas im Kindes- und Jugendalter (AGA), 2016. (available at: http://www.aga.adipositas-gesellschaft.de/index. php?id=9)

13 Cole TJ, Bellizzi MC, Flegal KM \& Dietz WH. Establishing a standard definition for child overweight and obesity worldwide: international survey. BMJ 2000320 1240-1243. (doi:10.1136/bmj.320.7244.1240)

14 Slaughter M, Lohmann T, Boileau R, Horswill C, Stillmann R \& Bemben D. Skinfold equations for estimation of body fatness in children and youth. Human Biology 199860 709-723.

15 Kulle AE, Reinehr T, Simic-Schleicher G, Hornig NC \& Holterhus PM. Determination of 17OHPreg and DHEAS by LCMSMS: impact of age, sex, pubertal stage and BMI on the Delta5-steroid-pathway. Journal of Clinical Endocrinology and Metabolism 2016102 232-241. (doi:10.1210/jc.2016-2849).

16 Vermeulen A, Verdonck L \& Kaufman JM. A critical evaluation of simple methods for the estimation of free testosterone in serum. Journal of Clinical Endocrinology and Metabolism 199984 3666-3672. (doi:10.1210/jcem.84.10.6079)

17 Ornstein RM, Copperman NM \& Jacobson MS. Effect of weight loss on menstrual function in adolescents with polycystic ovary syndrome. Journal of Pediatric and Adolescent Gynecology 201124 161-165. (doi:10.1016/j.jpag.2011.01.002)

18 Moran LJ, Hutchison SK, Norman RJ \& Teede HJ. Lifestyle changes in women with polycystic ovary syndrome. Cochrane Database of Systematic Reviews 20112 CD007506.

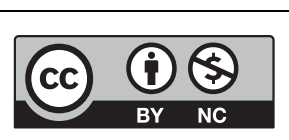

This work is licensed under a Creative Commons Attribution-NonCommercial 4.0 International License. 
19 Escobar-Morreale HF, Botella-Carretero JI, Alvarez-Blasco F, Sancho J \& San Millan JL. The polycystic ovary syndrome associated with morbid obesity may resolve after weight loss induced by bariatric surgery. Journal of Clinical Endocrinology and Metabolism 200590 6364-6369. (doi:10.1210/jc.2005-1490)

20 Bergh C, Carlsson B, Olsson JH, Selleskog U \& Hillensjo T. Regulation of androgen production in cultured human thecal cells by insulin-like growth factor I and insulin. Fertility and Sterility 199359 323-331. (doi:10.1016/S0015-0282(16)55675-1)

21 Singh A, Hamilton-Fairley D, Koistinen R, Seppala M, James VH, Franks S \& Reed MJ. Effect of insulin-like growth factor-type I (IGF-I) and insulin on the secretion of sex hormone binding globulin and IGF-I binding protein (IBP-I) by human hepatoma cells. Journal of Endocrinology 1990124 R1-R3. (doi:10.1677/joe.0.124R001)

22 Pinola P, Piltonen TT, Puurunen J, Vanky E, Sundstrom-Poromaa I, Stener-Victorin E, Ruokonen A, Puukka K, Tapanainen JS \& MorinPapunen LC. Androgen profile through life in women with polycystic ovary syndrome: a Nordic multicenter collaboration study. Journal of Clinical Endocrinology and Metabolism 2015100 3400-3407. (doi:10.1210/jc.2015-2123)

23 Remer T, Boye KR, Hartmann MF \& Wudy SA. Urinary markers of adrenarche: reference values in healthy subjects, aged 3-18 years. Journal of Clinical Endocrinology and Metabolism 200590 2015-2021. (doi:10.1210/jc.2004-1571)

24 Kim SH, Moon JY, Sasano H, Choi MH \& Park MJ. Body fat mass is associated with ratio of steroid metabolites reflecting 17,20-lyase activity in prepubertal girls. Journal of Clinical Endocrinology and Metabolism 2016101 4653-4660. (doi:10.1210/jc.2016-2515)

25 Biason-Lauber A, Zachmann M \& Schoenle EJ. Effect of leptin on CYP17 enzymatic activities in human adrenal cells: new insight in the onset of adrenarche. Endocrinology 2000141 1446-1454. (doi:10.1210/en.141.4.1446)

26 Mueller JW, Gilligan LC, Idkowiak J, Arlt W \& Foster PA. The regulation of steroid action by sulfation and desulfation. Endocrine Reviews 201536 526-563. (doi:10.1210/er.2015-1036)

27 Gingras S, Cote S \& Simard J. Multiple signal transduction pathways mediate interleukin-4-induced 3beta-hydroxysteroid dehydrogenase/ Delta5-Delta4 isomerase in normal and tumoral target tissues. Journal of Steroid Biochemistry and Molecular Biology 200176 213-225. (doi:10.1016/S0960-0760(00)00148-5)

28 Ibanez L, de ZF \& Potau N. Premature pubarche, ovarian hyperandrogenism, hyperinsulinism and the polycystic ovary syndrome: from a complex constellation to a simple sequence of prenatal onset. Journal of Endocrinological Investigation 199821 558-566. (doi:10.1007/BF03350781)

29 Gourgari E, Lodish M, Keil M, Sinaii N, Turkbey E, Lyssikatos C, Nesterova M, de la Luz Sierra M, Xekouki P, Khurana D, et al. Bilateral adrenal hyperplasia as a possible mechanism for hyperandrogenism in women with polycystic ovary syndrome. Journal of Clinical
Endocrinology and Metabolism 2016101 3353-3360. (doi:10.1210/ jc.2015-4019)

30 Ibanez L, Potau N, Marcos MV \& de ZF. Corticotropin-releasing hormone as adrenal androgen secretagogue. Pediatric Research 199946 351-353. (doi:10.1203/00006450-199909000-00018)

31 Kulle AE, Welzel M, Holterhus PM \& Riepe FG. Implementation of a liquid chromatography tandem mass spectrometry assay for eight adrenal C-21 steroids and pediatric reference data. Hormone Research in Paediatrics 201379 22-31. (doi:10.1159/000346406)

32 Gawlik A, Shmoish M, Hartmann MF, Malecka-Tendera E, Wudy SA \& Hochberg Z. Steroid metabolomic disease signature of nonsyndromic childhood obesity. Journal of Clinical Endocrinology and Metabolism 2016101 4329-4337. (doi:10.1210/jc.2016-1754)

33 Chen J, Shen S, Tan Y, Xia D, Xia Y, Cao Y, Wang W, Wu X, Wang H, Yi L, et al. The correlation of aromatase activity and obesity in women with or without polycystic ovary syndrome. Journal of Ovarian Research 20158 11. (doi:10.1186/s13048-015-0139-1)

34 Savas-Erdeve S, Keskin M, Sagsak E, Cenesiz F, Cetinkaya S \& Aycan Z. Do the anti-Mullerian hormone levels of adolescents with polycystic ovary syndrome, those who are at risk for developing polycystic ovary syndrome, and those who exhibit isolated oligomenorrhea differ from those of adolescents with normal menstrual cycles? Hormone Research in Paediatrics 201685 406-411. (doi:10.1159/000446111)

35 Durlinger AL, Gruijters MJ, Kramer P, Karels B, Kumar TR, Matzuk MM, Rose UM, de Jong FH, Uilenbroek JT, Grootegoed JA, et al. Anti-Mullerian hormone attenuates the effects of FSH on follicle development in the mouse ovary. Endocrinology 2001142 4891-4899. (doi:10.1210/en.142.11.4891)

36 La Marca A., Orvieto R, Giulini S, Jasonni VM, Volpe A \& De Leo V. Mullerian-inhibiting substance in women with polycystic ovary syndrome: relationship with hormonal and metabolic characteristics. Fertility and Sterility 200482 970-972. (doi:10.1016/j. fertnstert.2004.06.001)

37 Sartori C, Lazzeroni P, Merli S, Patianna VD, Viaroli F, Cirillo F, Amarri S \& Street ME. From placenta to polycystic ovarian syndrome: the role of adipokines. Mediators of Inflammation 201620164981916. (doi:10.1155/2016/4981916)

38 Ibanez L, Lopez-Bermejo A, Diaz M, Marcos MV \& de ZF. Early metformin therapy (age 8-12 years) in girls with precocious pubarche to reduce hirsutism, androgen excess, and oligomenorrhea in adolescence. Journal of Clinical Endocrinology and Metabolism 201196 E1262-E1267. (doi:10.1210/jc.2011-0555)

39 Bekx MT, Connor EC \& Allen DB. Characteristics of adolescents presenting to a multidisciplinary clinic for polycystic ovarian syndrome. Journal of Pediatric and Adolescent Gynecology 201023 7-10. (doi:10.1016/j.jpag.2009.04.004)

40 Rosenfield RL. Clinical review: identifying children at risk for polycystic ovary syndrome. Journal of Clinical Endocrinology and Metabolism 200792 787-796. (doi:10.1210/jc.2006-2012)

Received in final form 30 March 2017

Accepted 3 April 2017

Accepted Preprint published online 3 April 2017 http://www.endocrineconnections.org DOI: 10.1530/EC-17-0051

(c) 2017 The authors Published by Bioscientifica Ltd

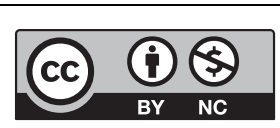

This work is licensed under a Creative Commons Attribution-NonCommercial 4.0 International License. 